\title{
Anti-CD3/Anti-BCMA Bispecific Monoclonal Antibody JNJ-64007957
}

National Cancer Institute

\section{Source}

National Cancer Institute. Anti-CD3/Anti-BCMA Bispecific Monoclonal Antibody/NL-

64007957. NCl Thesaurus. Code C136823.

\begin{abstract}
A bispecific humanized monoclonal antibody against human CD3, a T-cell surface antigen, and human B-cell maturation antigen (BCMA; TNFRSF17), a tumor-associated antigen (TAA) expressed on plasma cells, with potential antineoplastic activity. Upon administration, anti-CD3/anti-BCMA bispecific monoclonal antibody JNJ-64007957 binds to both CD3 on T-cells and BCMA expressed on malignant plasma cells. This results in the cross-linking of T-cells and tumor cells, and induces a potent cytotoxic T-lymphocyte $(C T L)$ response against BCMA-expressing plasma cells. BCMA, a member of the tumor necrosis factor receptor superfamily that is specifically overexpressed on malignant plasma cells, plays a key role in promoting plasma cell survival.
\end{abstract}

